\title{
Association between Life Stressors and Arterial Stiffness: The Finnish Retirement and Aging Study
}

\author{
Saana Karelius ${ }^{1,2, *}$, Jussi Vahtera ${ }^{3,4}$, Olli J. Heinonen ${ }^{5}$, Teemu J. Niiranen ${ }^{1,2,6, \dagger}$, Sari Stenholm ${ }^{2,3, \dagger}$ \\ ${ }^{1}$ Department of Internal Medicine, University of Turku, Turku 20014, Finland \\ ${ }^{2}$ Division of Medicine, Turku University Hospital, Turku, Finland \\ ${ }^{3}$ Department of Public Health, University of Turku and Turku University Hospital, Turku, Finland \\ ${ }^{4}$ Centre for Population Health Research, University of Turku and Turku University Hospital, Turku, Finland \\ ${ }^{5}$ Paavo Nurmi Centre \& Unit for Health and Physical Activity, University of Turku, Turku, Finland \\ ${ }^{6}$ Department of Public Health Solutions, Finnish Institute for Health and Welfare, Helsinki, Finland
}

\section{ARTICLE INFO}

\section{Article History}

Received 12 November 2020

Accepted 22 May 2021

Keywords

Ageing

blood pressure

cardiovascular disease

occupational health

stress

pulse wave velocity

\begin{abstract}
Objective: Besides traditional risk factors, other factors such as life stressors are linked with incident cardiovascular disease. However, the underlying mechanisms for this association remain mostly unknown. We studied the relation of life stressors (job strain, sleep loss due to worry, illness or death in family, financial difficulties and caregiving) and their accumulation with arterial stiffness, an independent predictor of cardiovascular disease.
\end{abstract}

Methods: 258 participants (mean age 62.4 years, 82\% women) from the Finnish Retirement and Aging Study underwent measurements for carotid-femoral Pulse Wave Velocity (PWV), a standard criterion for assessing arterial stiffness and responded to a survey inquiring life stressors. Using analysis of covariance, we estimated group means adjusted for age, gender, occupation, lifestyle factors and hypertension.

Results: Participants with recent illness or death in family $(8.04 \mathrm{~m} / \mathrm{s} ; 95 \%$ CI, 7.40-8.73 vs. $7.52 \mathrm{~m} / \mathrm{s}$; 95\% CI, 7.03-8.05) and financial difficulties $(8.65 \mathrm{~m} / \mathrm{s} ; 95 \%$ CI, 7.62-9.81 vs. $7.71 \mathrm{~m} / \mathrm{s} ; 95 \%$ CI, 7.21-8.24) had increased PWV compared to those who did not have exposed to these stressors independent of lifestyle factors, diabetes and systolic blood pressure. In addition, increasing number of life stressors demonstrated an association towards increased PWV ( $\geq 2$ stressors: $8.04 \mathrm{~m} / \mathrm{s} ; 95 \% \mathrm{CI}$, $7.42-8.72$ vs. 0 stressors $7.74 \mathrm{~m} / \mathrm{s} ; 95 \%$ CI, 7.23-8.28; $p$ for trend 0.27 ) but the association attenuated after adjusting for lifestyle factors, diabetes and systolic blood pressure.

Conclusion: Life stress was found to associate with higher arterial stiffness. Increased arterial stiffness could explain some of the increased cardiovascular disease risk related to life stressors.

\section{HIGHLIGHTS}

- Arterial stiffness was assesed as carotid-femoral pulse wave velocity.

- Illness or death in the family were associated with an increased arterial stiffness.

- Financial difficulties were associated with an increased arterial stiffness.

- A trend toward increasing number of stressors and higher arterial stiffness exists.

(C) 2021 The Authors. Publishing services by Atlantis Press International B.V. This is an open access article distributed under the CC BY-NC 4.0 license (http://creativecommons.org/licenses/by-nc/4.0/).

\section{INTRODUCTION}

Cardiovascular Disease (CVD) is a leading cause of mortality worldwide. Conventional risk factors, e.g., smoking, hypercholesterolemia and high blood pressure has been shown to partially

\footnotetext{
Corresponding author.Email: sekkar@utu.fi

${ }^{\dagger}$ The authors contributed equally to this work.

Peer review under responsibility of the Association for Research into Arterial Structure and Physiology

Data availability statement: Anonymized partial datasets of the Finnish Retirement and Aging Study are available by application with bona fide researchers with an established scientific record and bona fide organizations. For more information, please contact prof. Sari Stenholm (sari.stenholm@utu.fi).
}

explain the increased risk [1]. However, other CVD risk factors have also been identified, including exposure to stressors in private life or at work, such as stressful events and job strain [2]. Chronic stress resulting from occupational stressors has been associated with higher blood pressure in some studies, but the association between other life stressors and blood pressure is more complicated $[3,4]$.

The exact mechanisms through which mental strain and stress increase CVD risk remain obscure [5]. Increased arterial stiffness, most commonly measured using carotid-femoral Pulse Wave Velocity (PWV), is a strong predictor of future CVD events [6]. A few studies have examined the association of stressful life events, life stress and job strain with arterial stiffness [7-13]. However, 
since these studies have provided conflicting findings, were based on study samples that consisted mainly of young Asians, and have used non-standard methods for measuring PWV, further research on this topic is warranted.

The aim of this study was to examine the association of life stressors related to work and private life and their accumulation, with arterial stiffness, among late middle-aged persons.

\section{MATERIALS AND METHODS}

\subsection{Study Sample}

The study sample consisted of participants from the Finnish Retirement and Aging Study (FIREA). Details of the design and implementation of the FIREA study have been reported elsewhere [14]. The eligible population for the FIREA study cohort included all public sector employees with an estimated retirement date between 2014 and 2019 who worked in 2012 in Southwest Finland or in the nine selected cities or five hospital districts around Finland and who responded to at least one of the FIREA questionnaires $(N=6679)$. Participants were first contacted 18 months prior to their estimated retirement date by sending a questionnaire. After responding to the questionnaire, Finnish speaking participants, with estimated retirement date between 2017 and 2019, who lived in the Southwest Finland and were still working, were invited to participate in the clinical substudy $(n=773)$. Of them, 291 participated in the study between September 2015 and May 2018. We excluded 31 persons for not undergoing PWV measurements (due to refusal, cardiac arrhytmia, pacemaker, failed measurement or unknown reasons) and two persons for not answering the stressor questions, resulting on a final sample of 258 participants. The participants who took part in the PWV measurements reported less job strain, were physically more active, less often smokers, had lower body mass index as well as reported less hypertension and diabetes than survey participants indicating a healthier study population (Table 1).

The FIREA study was conducted in accordance with the Helsinki declaration and was approved by the Ethics Committee of Hospital District of Southwest Finland (ETMK: 84/1801/2014). All participants gave written informed consent.

\subsection{Assessment of Life Stressors}

Job strain was assessed with nine items measuring job control and five items measuring job demands derived from the Job Content Questionnaire [15]. The presence of job strain was defined as having high demands (above the median score of 3.2) and a low control (below the median score of 3.8) based on the FIREA surveys cut-off points. Sleep loss due to worry was obtained from the single question of General Health Questionnaire [16] in which participants were asked "Have you recently lost much sleep due to worry?" Participants were categorized into no ("Not at all" and "No more than usual") and yes ("Rather more than usual" and "Much more than usual"). Severe life events within the past 12 months (yes vs. no) were measured by the occurrence of severe illness or death in the family (spouse, child, parent or other) and severe financial difficulties were based on a question of having had severe financial problems within the past 12 months [yes vs. no (earlier or

Table 1 The selection of survey participants

\begin{tabular}{|c|c|c|c|c|c|}
\hline & \multicolumn{2}{|c|}{ Survey participants $(n=4649)$} & \multicolumn{3}{|c|}{ Participants who underwent PWV measurement $(n=258)$} \\
\hline & Mean (SD) & $N(\%)$ & Mean (SD) & $N(\%)$ & $p$-value \\
\hline Age & $62.5(1.2)$ & & $62.4(1.0)$ & & 0.03 \\
\hline Female & & $3797(82)$ & & $212(82)$ & 0.84 \\
\hline \multicolumn{6}{|l|}{ Life stressors } \\
\hline Job strain & & $1127(29)$ & & $55(22)$ & 0.02 \\
\hline Sleep loss because of worries & & $733(16)$ & & $40(16)$ & 0.89 \\
\hline Illness or death in the family & & $768(18)$ & & $44(18)$ & 0.99 \\
\hline Financial difficulties & & $165(4)$ & & $11(4)$ & 0.58 \\
\hline Caregiving & & $710(16)$ & & $43(17)$ & 0.53 \\
\hline Sum of life stressors & & & & & 0.92 \\
\hline 0 points & & $2207(47)$ & & $123(48)$ & \\
\hline 1 point & & $1614(35)$ & & $87(34)$ & \\
\hline 2 or more points & & $828(18)$ & & $48(19)$ & \\
\hline Occupational status & & & & & 0.08 \\
\hline Higher non-manual & & $1483(32)$ & & $95(37)$ & \\
\hline Lower non-manual & & $1395(30)$ & & $84(33)$ & \\
\hline Manual & & $1729(38)$ & & $79(30)$ & \\
\hline Self-reported body mass index & & & & & 0.04 \\
\hline Normal weight & & $1767(39)$ & & $109(43)$ & \\
\hline Overweight & & $1818(40)$ & & $106(42)$ & \\
\hline Obese & & $972(21)$ & & $37(15)$ & \\
\hline Low physical activity & & $1815(39)$ & & $64(25)$ & $<0.0001$ \\
\hline Heavy alcohol use & & $388(8)$ & & $29(11)$ & 0.11 \\
\hline Current smokers & & $496(11)$ & & $13(5)$ & 0.004 \\
\hline Self-reported hypertension & & $1660(39)$ & & $62(25)$ & $<0.0001$ \\
\hline Self-reported diabetes & & $428(10)$ & & $13(5)$ & 0.02 \\
\hline
\end{tabular}


not at all)]. Caregiving was assessed with the following question: "Do you provide care to a family member or relative, who is unable to take care of himself or herself because of age, illness or disability?" (giving a care for health children was discounted) (yes vs. no).

Based on these five life stressors (job strain, sleep loss due to worry, illness or death in family, financial difficulties and caregiving), the cumulative sum score was calculated by giving one point to each stressor, maximum points being 5. Participants were then categorized into three groups: 0,1 and 2 or more points.

\subsection{Carotid-femoral Pulse Wave Velocity}

Arterial stiffness was assessed using carotid-femoral PWV measurements (SphygmoCor PVx with MM3 electronics module and Millar tonometer), as previously described [17]. Briefly, three electrodes were attached to the body to record timing of cardiac $\mathrm{R}$-wave (time of pulse wave departure from the heart). The pulse wave measurements were made at the right femoral artery and right common carotid artery (time of pulse wave arrival at the distal arteries). Distance between manubrium sternum and registration points was measured to give a straight distance between registration points. Transit time was acquired from the time delay between the feet of two waveforms in relation to the R-wave of the simultaneously recorded electrocardiogram (ECG). PWV was then calculated by dividing the distance by the transit time $(\mathrm{m} / \mathrm{s})$. PWV measurements were performed twice and averaged for statistical analyses. Participants were asked to avoid food, caffeine drinks and tobacco use for $3 \mathrm{~h}$ and alcohol for $24 \mathrm{~h}$ before the measurement. The PWV measurement happened in the different study visit than the blood pressure measurement.

\subsection{Covariates}

Occupational titles were obtained from the pension insurance institute for the municipal sector in Finland (Keva; based in Helsinki, Finland). To measure occupational status, occupational titles, coded according to the International Standard Classification of Occupation, were categorized to three groups: higher-grade nonmanual workers (e.g. teachers, physicians), lower-grade non-manual workers (e.g. registered nurses, technicians) and manual workers (e.g. cleaners, maintenance workers). Body Mass Index (BMI) was calculated from measured weight and height and categorised into normal weight $\left(<25.0 \mathrm{~kg} / \mathrm{m}^{2}\right)$, overweight $\left(25\right.$ to $\left.<30 \mathrm{~kg} / \mathrm{m}^{2}\right)$ and obese $\left(\geq 30 \mathrm{~kg} / \mathrm{m}^{2}\right)$. Physical activity was defined as average weekly hours of leisure-time physical activity and dichotomized into low ( $<14$ metabolic equivalent) and high groups based on the selfreported and pre-defined criteria [14]. Alcohol consumption was categorized according to the habitual frequencies of drinking beer, wine and spirits; heavy alcohol use (yes vs. no) was defined as $>16$ drinks/week for women and $>24$ drinks/week for men. Smoking status was categorized as non-smokers (never and former) and current smokers. A nurse who stayed in the room during the measurements measured blood pressure twice with a digital blood pressure monitor (Microlife WatchBP Office Central) from right arm in the sitting position. Diabetes was based on self-reported doctor diagnosed condition. In addition, participants were asked to list all their medication. We defined hypertension as the use of antihypertensive medication, systolic blood pressure $\geq 140 \mathrm{mmHg}$ or diastolic blood pressure $\geq 90 \mathrm{mmHg}$.

\subsection{Statistical Analyses}

Characteristics of the participants are provided as percentages for categorical variables and as mean values for continuous variables. We compared adjusted mean log-transformed PWV values between groups using analysis of covariance. Model 1 was performed using age and gender as covariates. Model 2 was additionally adjusted for occupational status, BMI, physical activity, alcohol consumption, diabetes and smoking. Model 3 was further adjusted for systolic blood pressure. Statistical analysis were performed with SAS software, version 9.4 (SAS Institute Inc., Cary, NC, USA).

\section{RESULTS}

The study sample characteristics are shown in Table 2 . The majority of the participants were women $(N=212 ; 82 \%)$ and mean age was $62.4 \pm 1.0$ years. Mean PWV was $7.8 \pm 1.3 \mathrm{~m} / \mathrm{s} .22 \%$ of the participants had job strain, $16 \%$ reported sleep loss due to worry, $18 \%$ had recent illness or death in the family, $4 \%$ had recent financial difficulties and $17 \%$ reported caregiving. $48 \%$ had none life stressors while $19 \%$ had two or more.

Table 3 shows the association between individual and cumulative life stressors and PWV. In the age and gender adjusted model (Model 1), PWV was higher among participants with recent

Table 2 Characteristics of the study participants $(n=258)$

\begin{tabular}{lcc}
\hline & $n(\%)$ & Mean (SD) \\
\hline Age, years & $212(82)$ & $62.4(1.0)$ \\
Women & & $7.8(1.3)$ \\
PWV, m/s & & $137.9(17.7)$ \\
Blood pressure from the right hand & & \\
Life stressors & $55(22)$ \\
$\quad$ Job strain & $40(16)$ \\
$\quad$ Sleep loss due to worry & $44(18)$ \\
Illness or death in the family & $11(4)$ \\
Financial difficulties & $43(17)$ \\
Caregiving & & \\
Sum of life stressors & $123(48)$ \\
$\quad$ p points & $87(34)$ \\
1 point & $48(19)$ \\
2 or more points & \\
Occupational status & $95(37)$ \\
$\quad$ Higher non-manual & $84(33)$ \\
Lower non-manual & $79(31)$ \\
$\quad$ Manual & \\
Body mass index & $106(41)$ \\
$\quad$ Normal weight $\left(<25 \mathrm{~kg} / \mathrm{m}^{2}\right)$ & $110(43)$ \\
Overweight $\left(25\right.$ to $\left.<30 \mathrm{~kg} / \mathrm{m}^{2}\right)$ & $42(16)$ \\
Obese $\left(\geq 30 \mathrm{~kg} / \mathrm{m}^{2}\right)$ & $64(25)$ \\
Low physical activity & $29(11)$ \\
Heavy alcohol use & $13(5)$ \\
Current smokers & $116(45)$ \\
Hypertension & & \\
Self-reported diabetes & & \\
\hline
\end{tabular}


Table 3 Adjusted mean levels of pulse wave velocity $(\mathrm{m} / \mathrm{s})$ in groups by individual and cumulative life stressors

\begin{tabular}{|c|c|c|c|c|}
\hline \multirow{2}{*}{ Life stressor } & \multirow{2}{*}{$n(\%)$} & Model 1 & \multirow{2}{*}{$\frac{\text { Model } 2}{\text { Mean }(95 \% \text { CI })}$} & \multirow{2}{*}{$\frac{\text { Model } 3}{\text { Mean }(95 \% \text { CI }}$} \\
\hline & & Mean $(95 \% \mathrm{CI})$ & & \\
\hline \multicolumn{5}{|l|}{ Job strain } \\
\hline No & $197(78)$ & $7.70(7.48-7.92)$ & $7.78(7.25-8.34)$ & $7.78(7.26-8.34)$ \\
\hline Yes & $55(22)$ & $7.82(7.48-8.19)$ & $7.80(7.16-8.48)$ & $7.82(7.19-8.50)$ \\
\hline$p$-value & & 0.50 & 0.94 & 0.86 \\
\hline \multicolumn{5}{|c|}{ Sleep loss due to worry } \\
\hline No & $218(85)$ & $7.69(7.49-7.90)$ & $7.78(7.28-8.33)$ & $7.79(7.29-8.33)$ \\
\hline Yes & $40(16)$ & $7.76(7.37-8.18)$ & $7.77(7.13-8.46)$ & $7.76(7.13-8.45)$ \\
\hline$p$-value & & 0.74 & 0.94 & 0.91 \\
\hline \multicolumn{5}{|c|}{ Illnessess or death in family } \\
\hline No & $200(82)$ & $7.63(7.43-7.82)$ & $7.48(6.99-8.01)$ & $7.52(7.03-8.05)$ \\
\hline Yes & $44(18)$ & $8.09(7.69-8.51)$ & $7.93(7.29-8.62)$ & $8.04(7.40-8.73)$ \\
\hline$p$-value & & 0.02 & 0.042 & 0.02 \\
\hline \multicolumn{5}{|c|}{ Financial difficulties } \\
\hline No & $243(96)$ & $7.68(7.49-7.88)$ & $7.70(7.20-8.24)$ & $7.71(7.21-8.24)$ \\
\hline Yes & $11(4)$ & $8.39(7.61-9.24)$ & $8.66(7.61-9.84)$ & $8.65(7.62-9.81)$ \\
\hline$p$-value & & 0.07 & 0.056 & 0.06 \\
\hline \multicolumn{5}{|l|}{ Caregiver } \\
\hline No & $209(83)$ & $7.69(7.49-7.90)$ & $7.75(7.23-8.32)$ & $7.78(7.26-8.33)$ \\
\hline Yes & $43(17)$ & $7.74(7.35-8.16)$ & $7.70(7.07-8.39)$ & $7.62(7.01-8.29)$ \\
\hline$p$-value & & 0.80 & 0.82 & 0.50 \\
\hline \multicolumn{5}{|l|}{ Stress sum } \\
\hline 0 & $123(48)$ & $7.61(7.38-7.85)$ & $7.73(7.22-8.29)$ & $7.74(7.23-8.28)$ \\
\hline 1 & $87(34)$ & $7.71(7.42-8.00)$ & $7.75(7.18-8.36)$ & $7.74(7.18-8.34)$ \\
\hline$>1$ & $48(19)$ & $8.03(7.64-8.44)$ & $8.01(7.38-8.70)$ & $8.04(7.42-8.72)$ \\
\hline$p$ for trend & & 0.06 & 0.30 & 0.27 \\
\hline
\end{tabular}

Model 1: adjusted for age and gender. Model 2: Model 1 and additionally adjusted for occupational status, BMI, physical activity, alcohol consumption, smoking and diabetes. Model 3: Model 2 and additionally adjusted for systolic blood pressure.

illness or death in the family and borderline higher among participants with recent financial difficulties compared to those without these events, 8.09 (95\% CI 7.69-8.51) vs. 7.63 (95\% CI 7.43-7.82); 8.39 (95\% CI 7.61-9.24) vs. 7.68 (95\% CI 7.49-7.88). No significant associations were observed for job strain, sleep loss due to worry, or caregiving with PWV. In the lifestyle factors and diabetes adjusted model (Model 2), PWV both in participants with recent illness or death in family and recent financial difficulties proved to be borderline significant higher compared to those without these risks, 7.93 (95\% CI 7.29-8.62) vs. 7.48 (95\% CI 6.99-8.01); 8.66 (95\% CI 7.61-9.84) vs. 7.70 (95\% CI 7.20-8.24). Moreover, in the Model 3, additionally adjusted for systolic blood pressure, participants with resent illness or death in family had significantly higher PWV compared to those without this risk, 8.04 (95\% CI 7.40-8.73) vs. 7.52 (95\% CI 7.03-8.05). Again, participants with recent financial difficulties showed borderline significantly higher PWV values compared to participants without recent financial difficulties, 8.65 (95\% CI 7.62-9.81) vs. 7.71 (95\% CI 7.21-8.24). A trend toward higher PWVs with increasing number of stressors was observed, but adjustment for lifestyle factors, diabetes and systolic blood pressure attenuated the association.

\section{DISCUSSION}

We observed an increased arterial stiffness among the individuals who reported occurrence of a recent illness or death in the family or financial difficulties within the past 12 months. Other studied life stressors, such as job strain, sleep loss due to worry and caregiving were not associated with arterial stiffness. We observed a borderline association between increasing number of life stressors with higher PWV, which was however attenuated after taking into account lifestyle factors, diabetes and systolic blood pressure.

To the best of our knowledge, the association between recent death or illness in family and greater arterial stiffness has not been previously reported. However, this finding is in line with the earlier finding on the increased risk of myocardial infarct in parents that had faced a death of a child [18]. We also observed equivocal evidence of an association between higher PVW and multiple life stressors and so far very little research on the combined impact of multiple stressors on CVD risk has been conducted [19]. These findings are plausible, since it has been debated that stress can result in cardiovascular diseases via dysregulation of the hypothalamic-pituitary axis, inflammation and sympathetic nervous system activation [20].

From clinical point of view, it has been earlier concluded that a change of $1 \mathrm{~m} / \mathrm{s}$ in carotid-femoral PWV corresponds to $12 \%$ increased risk to cardiovascular events [21]. Moreover, the mortality to cardiovascular diseases increases by $9 \%$ while carotidfemoral PWV increases by $1 \mathrm{~m} / \mathrm{s}$ [21]. While in our study between group differences did not reach that level it can be assumed that the higher PWV values can lead to unfavourable consequences. However, it has to be regarded that the PWV values were overall at a good level in this study population consisting of relatively healthy aging workers. 
The strengths of our study include having a relatively large number of participants with varying occupational backgrounds as earlier studies have concluded that manual workers may be more vulnerable to job strain induced high blood pressure [22], a strong correlate of arterial stiffness. Moreover, we used carotid-femoral PWV, the noninvasive gold standard for measuring PWV [23], in contrast to previous studies that used a wide scale of non-conventional methods for assessing arterial stiffness (augmentation index, brachialankle pulse wave velocity and finger photoplethysmogram) [7,8,10-12].

The present study has also limitations. First, aging workers are a selected group of individuals that may be somewhat healthier than the general population. This was also expressed by the relatively low mean PWV of the study sample $(7.8 \mathrm{~m} / \mathrm{s})$. Moreover, the participation rate was relatively low leading to healthier study population compared to the FIREA survey participants. This may have distorted the association between life stressors and PWV. Despite we were able to control for the role of blood pressure and lifestyle factors in the association between stressors and PWV, residual confounding due to unmeasured confounders remains an issue in an observational study. Finally, as an observational study this study is primaly hypothesis-generating and risk of false positive findings also remains.

\section{CONCLUSION}

Recent death or illness in family and recent financial difficulties associate with increased PWV even after controlling for systolic blood pressure. Moreover, the PWV showed an elevating trend toward increasing stressors. Increased arterial stiffness could explain some of the cardiovascular disease risk related to life stressors. Future studies are warranted to examine the association of different stressors and arterial stiffness in more general populations.

\section{CONFLICTS OF INTEREST}

The authors declare they have no conflicts of interest.

\section{AUTHORS' CONTRIBUTION}

SK, TJN and SS conceived and designed the work. SS contributed to data acquisition. SK, TJN and SS analyzed, and interpreted the data. SK, TJN and SS drafted the manuscript. JV and OJH revised the manuscript critically for important intellectual content.

\section{FUNDING}

The funders had no role in the study design, the collection, analysis and interpretation of the data, the writing of the report, or the decision to submit the paper for publication.

\section{ACKNOWLEDGMENTS}

This work was supported by grants from the Urmas Pekkala Foundation and Turku University Foundation (for SK), the
Hospital District of Southwest Finland (for TJN), the Academy of Finland (grant number 286294, 294154, 332030 and 319246 for SS; and 321351 for TJN), the Emil Aaltonen Foundation (for TJN), the Finnish Medical Foundation (for TJN), the Finnish Foundation for Cardiovascular Research (for TJN) and the Paavo Nurmi Foundation (for TJN).

\section{REFERENCES}

[1] GBD 2015 Risk Factors Collaborators. Global, regional, and national comparative risk assessment of 79 behavioural, environmental and occupational, and metabolic risks or clusters of risks, 1990-2015: a systematic analysis for the Global Burden of Disease Study 2015. Lancet 2016;388:1659-724.

[2] Kivimäki M, Nyberg ST, Batty GD, Fransson EI, Heikkilä K, Alfredsson L, et al. Job strain as a risk factor for coronary heart disease: a collaborative meta-analysis of individual participant data. Lancet 2012;380:1491-7.

[3] Hassoun L, Herrmann-Lingen C, Hapke U, Neuhauser H, Scheidt-Nave C, Meyer T. Association between chronic stress and blood pressure: findings from the German Health Interview and Examination Survey for Adults 2008-2011. Psychosom Med 2015;77:575-82.

[4] Sparrenberger F, Cichelero FT, Ascoli AM, Fonseca FP, Weiss G, Berwanger $\mathrm{O}$, et al. Does psychosocial stress cause hypertension? A systematic review of observational studies. J Hum Hypertens 2009;23:12-19.

[5] Steptoe A, Kivimäki M. Stress and cardiovascular disease. Nat Rev Cardiol 2012;9:360-70.

[6] Ben-Shlomo Y, Spears M, Boustred C, May M, Anderson SG, Benjamin EJ, et al. Aortic pulse wave velocity improves cardiovascular event prediction: an individual participant meta-analysis of prospective observational data from 17,635 subjects. J Am Coll Cardiol 2014;63:636-46.

[7] Bomhof-Roordink H, Seldenrijk A, van Hout HPJ, van Marwijk HWJ, Diamant M, Penninx BWJH. Associations between life stress and subclinical cardiovascular disease are partly mediated by depressive and anxiety symptoms. J Psychosom Res 2015;78:332-9.

[8] Kaewboonchoo O, Sembajwe G, Li J. Associations between job strain and arterial stiffness: a large survey among enterprise employees from Thailand. Int J Environ Res Public Health 2018;15:659.

[9] Michikawa T, Nishiwaki Y, Nomiyama T, Uemura T, O’Uchi T, Sakurai H, et al. Job strain and arteriosclerosis in three different types of arteries among male Japanese factory workers. Scand J Work Environ Health 2008;34:48-54.

[10] Otsuka T, Kawada T, Ibuki C, Kusama Y. Relationship between job strain and radial arterial wave reflection in middle-aged male workers. Prev Med 2009;49:260-4.

[11] Utsugi M, Saijo Y, Yoshioka E, Sato T, Horikawa N, Gong Y, et al. Relationship between two alternative occupational stress models and arterial stiffness: a cross-sectional study among Japanese workers. Int Arch Occup Environ Health 2009;82:175-83.

[12] Nomura K, Nakao M, Karita K, Nishikitani M, Yano E. Association between work-related psychological stress and arterial stiffness measured by brachial-ankle pulse-wave velocity in young Japanese males from an information service company. Scand J Work Environ Health 2005;31:352-9. 
[13] Slepecky M, Kotianova A, Prasko J, Majercak I, Gyorgyova E, Kotian M, et al. Which psychological, psychophysiological, and anthropometric factors are connected with life events, depression, and quality of life in patients with cardiovascular disease. Neuropsychiatr Dis Treat 2017;13:2093-104.

[14] Leskinen T, Pulakka A, Heinonen OJ, Pentti J, Kivimäki M, Vahtera J, et al. Changes in non-occupational sedentary behaviours across the retirement transition: the Finnish Retirement and Aging (FIREA) study. J Epidemiol Community Health 2018; 72:695-701.

[15] Karasek R, Brisson C, Kawakami N, Houtman I, Bongers P, Amick B. The Job Content Questionnaire (JCQ): an instrument for internationally comparative assessments of psychosocial job characteristics. J Occup Health Psychol 1998;3:322-55.

[16] Goldberg DP. The detection of psychiatric illness by questionnaire; a technique for the identification and assessment of non-psychotic psychiatric illness. London: Oxford University Press; 1972.

[17] Lindroos AS, Johansson JK, Puukka PJ, Kantola I, Salomaa V, Juhanoja EP, et al. The association between home vs. ambulatory night-time blood pressure and end-organ damage in the general population. J Hypertens 2016;34:1730-7.
[18] Li J, Hansen D, Mortensen PB, Olsen J. Myocardial infarction in parents who lost a child: a nationwide prospective cohort study in Denmark. Circulation 2002;106:1634-9.

[19] Albert MA, Slopen N, Williams DR. Cumulative psychological stress and cardiovascular disease risk: a focused review with consideration of black-white disparities. Curr Cardiovasc Risk Rep 2013;7:318-25.

[20] McEwen BS. Protective and damaging effects of stress mediators. N Engl J Med 1998;338:171-9.

[21] Zhong Q, Hu MJ, Cui YJ, Liang L, Zhou MM, Yang YW, et al. Carotid-femoral pulse wave velocity in the prediction of cardiovascular events and mortality: an updated systematic review and meta-analysis. Angiology 2018;69:617-29.

[22] Landsbergis PA, Schnall PL, Pickering TG, Warren K, Schwartz JE. Lower socioeconomic status among men in relation to the association between job strain and blood pressure. Scand J Work Environ Health 2003;29:206-15.

[23] Townsend RR, Wilkinson IB, Schiffrin EL, Avolio AP, Chirinos JA, Cockcroft JR, et al. Recommendations for improving and standardizing vascular research on arterial stiffness: a scientific statement from the American Heart Association. Hypertension 2015;66:698-722. 